\title{
28 Research Square \\ Identification of a Potential Prognostic Signature based on Immune-related Genes in Primary Glioblastoma
}

\section{Zhaoming Zhou}

Southern Medical University

Mingyao Lai

Guangdong Sanjiu Brain Hospital

Jiayin $\mathrm{Yu}$

Southern Medical University https://orcid.org/0000-0001-9814-1503

Jiangfen Zhou

Guangdong Sanjiu Brain Hospital

Qingjun Hu

Guangdong Sanjiu Brain Hospital

Juan Li

Guangdong Sanjiu Brain Hospital

Hainan Li

Guangdong Sanjiu Brain Hospital

\section{Longhua Chen}

Southern Medical University Nanfang Hospital Department of Oncology

Lei Wen

Guangdong Sanjiu Brain Hospital

Meijuan Zhou ( $\square$ lkzmj@smu.edu.cn )

Southern Medical University https://orcid.org/0000-0003-4962-3164

Linbo Cai

Guangdong Sanjiu Brain Hospital

\section{Primary research}

Keywords: glioblastoma, immune-related genes, prognosis prediction, immune infiltration, TME

Posted Date: November 23rd, 2021

DOI: https://doi.org/10.21203/rs.3.rs-1078857/v1 
License: (c) (i) This work is licensed under a Creative Commons Attribution 4.0 International License. Read Full License 


\section{Abstract}

Background: Glioblastoma (GBM) is a common primary brain tumor with a high incidence in adults with malignant and fast-growing biological characteristics. In this study, we explore the immune-related prognosis markers of GBM at the mRNA level.

Methods: The sequencing data and clinical information of GBM patients were downloaded from The Cancer Genome Atlas (TCGA) and the Chinese Glioma Genome Atlas (CGGA). The differentially expressed genes (DEGs) were calculated between normal tissues from the Genotype-Tissue Expression (GTEx) database and tumor tissues from TCGA and CGGA. We obtained immune genes from the ImmProt database. The intersection of DEGs and immune genes were defined as the immune-related differential genes (IR-DEGs), based on which, survival associated IR-DEGs were determined by multivariate Cox regression analysis. The survival risk score (SRS) was determined for each sample with the top 6 prognostic associated IR-DEGs. One-year, two-year, and three-year potential survival were predicted by the prognosis prediction model established by multivariate and univariate Cox regression. In addition, we performed CIBERSORT in GBM patients with samples from TCGA cohort; association analysis was performed with prognostic IR-DEGs and immune cells. Furthermore, the influence of prognostic IR-DEGs on the brain tumor microenvironment (TME) was validated in single-cell sequencing analysis.

Results: We found 301 IR-DRGs in GBM primary tumor compared with normal tissue, and 19 of them could predict the overall survival (OS) more accurately in GBM patients. Six IR-DEGs (PLAUR, TNFSF14, CTSB, SOCS3, PTX3, and FCGR2B) were selected to construct the SRS, with which, GBM patients were divided into two different groups which combined with high and low risk. The SRS was found to be an independent prognostic factor for GBM and could predict GBM patients' possible survival with an acceptable efficiency. Moreover, the expression of 6 IR-DEGs and their co-expressed IR-DEGs could influence TME and were associated with GBM prognosis.

Conclusions: This study identified a potential immune prognostic signature of glioblastoma, which could enhance the prognosis prediction ability for GBM patients. The immune-related-genes in the TME could potentially benefit the immunotherapy development for GBM patients.

\section{Introduction}

Glioblastoma (GBM) is a common primary brain tumor with a high incidence in adults with malignant and fast-growing biological characteristics. Due to the dismal prognosis and the impact on life quality of life and cognitive function, GBM has become one of the most feared diseases. Remarkably, the median survival of glioblastoma is $9-15$ months and the 5-year survival rate of glioblastoma is $5.4 \%[1]$. Currently, the best treatment strategies available are maximum safe resection plus adjuvant chemoradiotherapy in glioblastoma patients. What's more, tumor-treating fields (TTF) with adjuvant temozolomide can also prolong GBM patient survival[1, 2]. However, primary glioblastomas will always recur due to aggressive 
and treatment-resistant recurrences, because of poor standard treatment for recurrent glioblastoma and finally the patients will quickly die with a short living time[3]. Extensive studies of immune checkpoint inhibitors (ICls) therapy, especially anti-programmed cell death protein-1 (PD-1)/programmed death ligand-1 (PD-L1) and anti-cytotoxic T lymphocyte-associated protein 4 (CTLA-4), have been conducted on primary and recurrent GBM in clinical research and were unsuccessful[4, 5]. Prognostic factors of GBM are related to age, performance status, tumor grade, specific biomarkers (MGMT methylation, mutation of IDH1, IDH2 or TERT, 1p19q co-deletion, overexpression of EGFR, etc.) and the extent of resection[6, 7]. Up to now, the existing evaluation markers cannot cover all the disease information of GBM patients. Thus, there is an immediate requirement for effective prognostic factors to help predict the prognosis evaluation of GBM.

TME plays a vital role in tumor growth and invasion, and ultimately affects the patient's prognosis. TME in GBM contains a variety of immune cell types, including tumor-associated macrophages (TAM), regulatory $T$ cells (Tregs), microglia, monocyte and bone marrow-derived suppressor cells[8, 9]. The proinflammatory cytokines such as IL-12, IL-18, and IFN- $y$ in TME of GBM were significantly reduced, while the soluble inhibitory molecules (interleukin 10, VEGF and beta transforming growth factor) were enriched[10,11]. Under certain circumstances, the complement system plays a key and complex role. It can help swallow antibody-coated tumor cells, induce a series of inflammatory responses, and help immune cells fight infection and maintain homeostasis, or hinder anti-tumor T cell responses that favor tumor progression[12]. Complement proteins exist in TME and tumor cells have the capacity to produce in situ[13]. C3a produced by tumor cells promotes immunosuppressive TME by acting on TAMs[14]. However, the imbalance between complement activation and inhibition will influence tumor development at different stages and finally influence tumor progression and invasion[15]. Various studies have reported that complement is activated in GBM, and tumor growth in situ is significantly reduced after the inhibition of the complement system in a cascade[15, 16]. However, the harmful impact on the prognosis of GBM remains to be confirmed.

Therefore, in order to identify the immune-related prognostic gene signature, we used differential gene analysis in The Atlas Cancer Genome (TCGA) GBM dataset and Chinese Glioma Genome Atlas (CGGA) dataset, intersection with ImmProt database, to screen 301 IR-DEGs. Then, filtered by Kaplan Meier survival analysis, the potential genes were applied to the multivariate analysis combining clinicalpathological features and gene expression. Six prognostic IR-DEGs (PLAUR, TNFSF14, CTSB, SOCS3, PTX3, FCGR2B) were selected. The prognosis model integrating six IR-DEGs was established to optimize the prognosis evaluation. The prognosis model was validated in an independent GBM cohort from CGGA. To identify the impact of prognostic IR-DEGs on TME, a total of 22 types of infiltrating immune cells in the 166 patients from TCGA divided into two different groups were estimated in CIBERSORT. The results were further verified in single-cell sequencing analysis. The regulatory network was constructed based on prognostic IR-DEGs and their co-expressed genes. The study provides a piece of benefits information that will guide clinical immunotherapy strategies. Moreover, the prognostic IR-DEGs could act as therapeutic targets for clinical immunotherapy of GBM. 


\section{Material And Method}

\section{Samples and Data acquisition}

We obtained 171 mRNA expression files with FPKM normalized from The Cancer Genome Atlas (TCGA, https://tcga-data.nci.nih.gov/tcga/), included 5 normal samples and 166 glioblastoma samples. We downloaded both GBM clinical samples from UCSC Xena (http://xena.ucsc.edu/), in which 107 patients have completed survival information and clinicopathological features. 129 cases of mRNA expression were downloaded from CGGA (http://www.cgga.org.cn/) for validation and 86 of them had complete survival information and clinicopathological features. We got data for the single cell GBM analysis was obtained from the Gene Expression Omnibus (GEO, https://www.ncbi.nlm.nih.gov/geo/), in which 2,343 cells from tumor cores and 1,246 cells from peripheral regions, During the process, the reading depth of $10 \times$ genomics was on the basis of Illumina NextSeq 500 , the accession number is GSE84465.

\section{Differential analysis and GBM sc-RNA data processing}

We used the R package "limma" (R version 4.0.2) to analyze differential gene expression. Genes with |logFCl (fold change) $>0.75$ and FDR $<0.05$ were considered as differential one. We got 5927 DEGs from the TCGA dataset, 4770 DEGs from the CGGA dataset, and 1793 immune-related genes from the ImmPort database (https://www.immport.org/). Next, we intersected each other and obtained 301 IR-DEGs in both TCGA and CGGA datasets. The statistical analysis was done in the scRNA-seq data, using the "Seurat R" package as quality control. According to the composition patterns of the marker genes in the singleR package, we determined and annotated different cell clusters on the basis of verification and correction from the CellMarker database[17].

\section{Identification of prognostic IR-DEGs}

Kaplan- Meier survival analysis was done by "survival" package in R to screen the IR-DEGs, which were significantly associated with the overall survival (OS) of the 107 samples from the TCGA dataset. To further determine the prognostic genes, all the information of 19 OS-related genes with $P<0.05$ were analyzed by multivariate cox regression analysis included clinical features such as age, gender, karnofsky performance score, radiation therapy and IDH mutation status. Then, we performed ROC analysis to screen the best prognostic genes, selecting six of them.

\section{Construction and evaluation of prognostic risk model}

The risk model was constructed on the ground of the six prognostic IR-DEGs. We used the following formula to calculate the risk score in each patient:

$$
\text { riskscore }=\sum_{i=1}^{n} \operatorname{Coef}_{i} * x_{i}
$$

where $\mathrm{Coef}_{i}$ mean the regression coefficient, calculated by the multivariate Cox regression, and $x_{i}$ represents the expression level of IR-DEG. GBM patients were divided into low-risk and high-risk groups according to the value of median risk score. Kaplan-Meier survival curve was charted to evaluate the OS 
of patients in two groups. The difference between the two groups was evaluated by a two-sided log-rank test. The ROC curve within 1, 3 and 5 years was plotted according to the "survivalROC" R package to assess the predictive accuracy of the risk model. To make clear that whether the risk score maybe an independent prognostic factor out of other clinicopathological parameters, we used multivariate Cox regression and univariate Cox regression analysis in TCGA testing cohort and CGGA validation cohort, Pvalue $<0.05$ was considered statistically significant.

\section{Tumor-Infiltrating Immune Cells in high- and low- risk group}

Immune cells were classified into 22 types such as T cells, B cells, plasma cells, NK cells, and myeloid subsets by CIBERSORT. According to the signature gene expression profile of the high-throughput array of RNA-sequencing data, the way of CIBERSORT mainly relied on the LM22 signature matrix[17]. Thus, we use CIBERSORT to analyze the mRNA expression matrix including 83 low-risk and 83 high-risk of glioblastoma samples from the TCGA dataset. Meanwhile we calculated the p-value for the deconvolution in each sample by the Monte Carlo sampling method. Finally, the relationship between IRDEGs and immune cells was uncovered through Person's correlation analysis, and the comprehensive connection of IR-DEGs and tumor infiltrating immune cells were represented, which was performed in TIMER2.0 (http://timer.cistrome.org/).

\section{Results}

\section{Identification of prognostic IR-DEGs}

The workflow chart of this study was shown in Figure 1. To investigate the immune-related-genes that might influence the prognosis of GBM. We obtained sequencing information with clinical data of 166 GBM samples from the TCGA dataset and 86 samples from CGGA, including RNA-seq (FPKM and counts format). Combined with normal samples from the GTEx dataset. Differential analysis was performed in normal tissues and tumor tissues with the filter condition FDR $<0.05,|\log F C|>1.5$ (Fig. 2a, 2b). And then, the overlap in Venn diagram of the intersection between differentially expressed genes in TCGA and CGGA and immune related genes in the Immprot dataset contained 301 IR-DEGs (Fig. 2c). Filtered by Kaplan-Meier survival analysis $(p<0.05), 19$ survival related IR-DEGs were determined (Table 1$)$. Then, those 19 IR-DEGs were applied to multivariate analysis combined with clinical-pathological features and gene expression, and six prognostic IR-DEGs (PLAUR, TNFSF14, CTSB, SOCS3, PTX3, FCGR2B) were selected for further analysis (Table 1). 
Table 1

Multivariate analysis and Kaplan-Meier survival analysis of prognostic genes

\begin{tabular}{|llllll|}
\hline Gene & KM & HR & HR.95L & HR.95H & coxPvalue \\
\hline SOCS3 & 0.023841 & 1.009035 & 1.004339 & 1.013752 & 0.000157 \\
\hline NMB & 0.000435 & 0.997357 & 0.994871 & 0.999848 & 0.037619 \\
\hline PLTP & 0.028617 & 1.001091 & 1.000102 & 1.002081 & 0.030545 \\
\hline PTX3 & 0.035228 & 1.008785 & 1.004178 & 1.013413 & 0.00018 \\
\hline RETN & 0.006169 & 1.147827 & 1.041132 & 1.265457 & 0.00561 \\
\hline CTSB & 0.000718 & 1.001569 & 1.000642 & 1.002497 & 0.000908 \\
\hline BMP2 & 0.032426 & 0.943501 & 0.906264 & 0.982269 & 0.004644 \\
\hline PLAUR & 0.009326 & 1.046651 & 1.024068 & 1.069731 & $4.19 E-05$ \\
\hline IFI30 & 0.007827 & 1.328068 & 1.050558 & 1.678883 & 0.017675 \\
\hline GRN & 0.000152 & 1.006865 & 1.002618 & 1.011131 & 0.001514 \\
\hline NRG1 & 0.018258 & 1.812052 & 1.303629 & 2.518761 & 0.000403 \\
\hline FCGR2B & 0.040158 & 1.097549 & 1.021167 & 1.179644 & 0.011436 \\
\hline RAC2 & 0.040014 & 1.049474 & 1.013386 & 1.086848 & 0.006836 \\
\hline SDC1 & 0.01936 & 1.011579 & 1.000525 & 1.022756 & 0.040016 \\
\hline IKBKE & 0.020406 & 1.132907 & 1.012126 & 1.2681 & 0.030043 \\
\hline TNFSF14 & 0.00298 & 1.291905 & 1.106777 & 1.507998 & 0.001172 \\
\hline IL13RA1 & 0.047883 & 1.014497 & 1.00001 & 1.029194 & 0.049838 \\
\hline CCL2 & 0.018237 & 1.002737 & 1.000167 & 1.005314 & 0.036848 \\
\hline CTSL & 0.002139 & 1.002775 & 1.000374 & 1.005183 & 0.023479 \\
\hline
\end{tabular}

\section{IR-DEGs based Risk score was an independent prognostic factor for GBM}

To optimize the prognosis evaluation system, we established prognosis model that integrated six prognostic IR-DEGs from GBM patients. In each sample, we calculated the risk score on the basis of the coefficients of the six predictive genes (PLAUR, TNFSF14, CTSB, SOCS3, PTX3, FCGR2B). According to the median risk score (Fig. 3a, 3b), we divided patients into high- and low-risk subgroups (Fig. 3a, 3b). Heatmap showed that the expression of the six prognostic IR-DEGs were different between the high-risk and low-risk group. The number of dead patients increased gradually as the risk score rose (Fig. 3a, 3b). 
Moreover, the Kaplan Meier survival curve showed that the OS between high- and low-risk groups was significantly different (Fig. 3c, 3d). The prognostic model showed higher accuracy in predicting 3-year and 5-year OS of GBM in TCGA and CGGA dataset, according to time-dependent ROC curve (Fig. 3c, 3d). To evaluate the independence of the prognostic model, we did univariate and multivariate Cox regression analyses in the TCGA dataset, and validated the result in an independent GBM cohort from CGGA. As shown in Table 2, the risk score was remarkably correlated with overall survival (OS) (HR=1.573, 95\% $\mathrm{Cl}=1.148-2.156, \mathrm{P}=0.005)$ in univariate analysis. The multivariate analysis illustrated that the risk score was an independent prognostic indicator $(\mathrm{HR}=1.566,95 \% \mathrm{Cl}=1.133-2.166, \mathrm{P}=0.007)$. In summary, these results showed that the risk score on the ground of prognostic IR-DEGs was an independent prognostic factor (Fig. 3c, 3d). 
Table 2

Univariate and multivariate analysis of risk in TCGA and CGGA dataset

\begin{tabular}{|c|c|c|c|c|}
\hline & $\begin{array}{l}\text { Univariate } \\
\text { analysis }\end{array}$ & & $\begin{array}{l}\text { Multivariate } \\
\text { Analysis }\end{array}$ & \\
\hline & $\mathrm{HR}(95 \% \mathrm{Cl})$ & $\begin{array}{l}P \\
\text { value }\end{array}$ & $\mathrm{HR}(95 \% \mathrm{Cl})$ & $\begin{array}{l}P \\
\text { value }\end{array}$ \\
\hline \multicolumn{5}{|l|}{ TCGA GBM testing set $(n=107)$} \\
\hline Age & $\begin{array}{l}1.233(0.908- \\
1.674)\end{array}$ & 0.179 & & \\
\hline Karnofsky_perfomance_score & $0.799(0.57-1.12)$ & 0.193 & & \\
\hline Radiation_therapy & $\begin{array}{l}0.344(0.208- \\
0.567)\end{array}$ & $<0.001$ & $0.329(0.197-0.55)$ & $<0.001$ \\
\hline Gender & $1.06(0.767-1.465)$ & 0.722 & & \\
\hline IDH_mutation_status & $\begin{array}{l}0.435(0.192- \\
0.986)\end{array}$ & 0.046 & $0.544(0.237-1.249)$ & 0.151 \\
\hline Risk & $\begin{array}{l}1.573(1.148- \\
2.156)\end{array}$ & 0.005 & $1.566(1.133-2.166)$ & 0.007 \\
\hline \multicolumn{5}{|l|}{$\begin{array}{l}\text { CGGA GBM validation cohort } \\
(n=86)\end{array}$} \\
\hline Gender & $0.766(0.55-1.066)$ & 0.114 & & \\
\hline Age & $1.021(0.695-1.5)$ & 0.915 & & \\
\hline Radiation_therapy & $\begin{array}{l}0.682(0.425- \\
1.096)\end{array}$ & 0.114 & & \\
\hline Chemotherapy & $\begin{array}{l}0.547(0.348- \\
0.859)\end{array}$ & 0.009 & $0.576(0.365-0.91)$ & 0.018 \\
\hline IDH_mutation_status & $\begin{array}{l}0.575(0.357- \\
0.924)\end{array}$ & 0.022 & $0.616(0.379-1)$ & 0.05 \\
\hline 1p19q_codeletion_status & $\begin{array}{l}0.782(0.345- \\
1.772)\end{array}$ & 0.555 & & \\
\hline MGMTp_methylation_status & $\begin{array}{l}1.099(0.792- \\
1.525)\end{array}$ & 0.571 & & \\
\hline Risk & $\begin{array}{l}1.662(1.179- \\
2.343)\end{array}$ & 0.004 & $1.447(1.017-2.058)$ & 0.04 \\
\hline
\end{tabular}

\section{Identification of Tumor-Infiltrating Immune cells in two risk group}


To identify the difference of infiltrating immune cells from the high-risk and low-risk groups of GBM, we analyzed the compendium of 22 immune cell types that infiltrated in tumors, in TCGA dataset with CIBERSORT. The making-up of immune cells from 166 samples and the relationship between the infiltrating immune cells showed in Figure 4. Macrophages were enriched both in the high-risk and lowrisk groups (Fig. 4a). The share of monocytes was remarkably increased in the high-risk group, while the share of NK cells was significantly enriched in the low-risk group (Fig. 5b).These results showed an immunosuppressive microenvironment in high-risk group.

\section{Prognostic IR-DEGs promote immune-suppressive micro- environment}

The relationship between six prognostic IR-DEGs and 22 immune cells was performed by using Person's correlation analysis. The results indicated that the expression levels of 6 prognostic IR-DEGs were positively correlated with monocytes and neutrophils, and had negative correlation with NK cells (Fig. 5a, 5c). In single cell analysis, PLAUR and CTSB expressed high in macrophages, SOCS3 and FCGR2B expressed low in macrophages. However, TNFSF14 and PTX3 were not detected in any of the cell types (Fig. 6). Furthermore, we identified the co-expressed genes related to six prognostic IR-DEGs by using Person's correlation analysis, and applied them to GO and KEGG enrichment analysis. The results of GO analysis showed that the prognostic IR-DEGs can be enriched in biological processes including neutrophil degranulation, cell chemotaxis and macrophage activation (Fig. 7b). KEGG analysis showed that prognostic IR-DEGs were mainly enriched in TNF signaling pathway and complement and coagulation cascades (Fig. 7a). In general, prognostic IR-DEGs plays a vital role in promoting the immunosuppressive microenvironment.

\section{Construction of regulatory network}

In order to clarify the regulation mechanism of prognostic IR-DEGs, we performed regulatory network combined with prognostic IR-DEGs and their co-expressed genes, as well as the regulatory pathways. PTX3 and PLAUR related to complement and coagulation cascades, SOCS3 activated TNF signaling pathway, CTSB activated antigen processing and presentation, NF-kappa B signaling pathway regulated by TNFSF14, FCGR2B activated Fc gamma R-mediated phagocytosis (Fig. 8a). Moreover, the transcription factors regulatory network of six prognostic IR-DEGs is shown in Figure 8b. These regulatory mechanisms collectively mediated immunosuppressive TME and promote tumor progression, and finally affecting the prognosis of GBM patients.

\section{Discussion}

The accumulation analysis of data shows that the basis of the interplay between cancer cells and the innate immune system is a unified cohesion mechanism. According to our analysis of TCGA and CGGA data, we first identified 301 differential immune-related genes and then confirmed that PLAUR, TNFSF14, CTSB, SOCS3, PTX3 and FCGR2B were significantly correlated with prognosis in univariate and multivariate Cox regression. The risk score of the six prognostic DEGs has great predictive potential in 
estimating the survival rate of GBM patients and promoting immune-suppressive TME of GBM. Currently, the therapeutic strategies of glioblastoma have been advanced, but the 5 -year survival rate is $5.4 \%[1,19]$. Therefore, the immune micro-environment acts an essential factor in the growth of novel therapies and shapes the outcome of patients with glioblastoma, and the prognostic IR-DEGs can be the promising prognostic predictors and therapeutic targets in GBM.

Glioblastoma has mortal cues. Take for instance, youth, poor cure state and incomplete scope of removal are identified as poor prognostic factors $[1,19]$. In elder, the median survival is less than four months with the best suitable treatment[22]. On the molecular level, isocitrate dehydrogenase 1 (IDH-1), IDH-2 variation and MGMT methylation have a better assessment of prognosis[23]. IDH mutation status can be divided into two different subgroups, including IDH-wild-type glioblastoma and IDH-mutant-type glioblastoma. On the one hand, IDH-Wild-type glioblastoma corresponds to the development of primary glioblastoma. This type represents approximately $90 \%$ of glioblastoma patients and usually appears in older patients with more clinical aggression[24]. On the other, IDH-Wild-type glioblastoma originates from either one-up spread or primary astrocytoma. This type contains approximately $10 \%$ of all glioblastoma patients. The diagnosis mainly emerges in 44-years median ages with a better prognosis[25]. In our analysis, the IDH mutation status was related to the overall survival in line with the ending of univariate analysis, but that was not an independent prognostic factor according to the multivariate analysis in the TCGA dataset. The underlying reason might be that it predicts OS of glioblastoma cooperate with other elements.

In GBM microenvironment, macrophages were enriched in both high-risk and low-risk groups, and the diversity in infiltration between the two groups was not remarkable. However, the abundance ratio of NK cells was higher in the low-risk group than in the high-risk group. The prognostic IR-DEGs were positively correlated with macrophages and negatively correlated with NK cells. Macrophages are the primary immune cells in brain tumors, accounting for up to $30 \%$ of malignant tumor[26]. TAMs (tumorassociated macrophages and microglia) are categorized as tissue-resident microglia and bone marrowderived macrophages (BMDMs), that are sorted to the glioma environment. TAMs have immunosuppressive impacts by releasing a broad range of growth factors and cytokines in response to the material derived from cancer cells[27]. In pathological circumstances, encircling monocytes are recruited to the brain essence and cause BMDMs[28]. The accumulative of macrophages in and around glioma actively influence glioma growth and invasion. Researchers found that, the expression of MT1MMP has positive correlations with the increasing glioma malignancy grade[29]. Toll-like receptors (TLR) are indispensable in the process of mediating immunologic responses to pathogens[29]. In the GBM microenvironment, TLR2 is appointed as the main TLR to trigger the course of MT1-MMP upregulation[31]. In the regulatory network, the Toll-like receptor signaling pathway is activated by TLR2, the co-expressed gene of the prognostic IR-DEGs, and that could promote tumor growth.

NK cells, the effector lymphocytes of the innate immune system, have the ability to limit tumors growth and dissemination[32]. However, tumors may resist attacks from endogenous NK cells through several paths, leading to immune evasion. The activation course of NK cell is mediated by the CD16 receptor (also known as FCGR3A), and the FCGR3A binds the constant region (Fc) of immunoglobulins to inflict 
heavy casualties on the antibody-coated cells[33]. Activating NK cell receptors and triggering optimal NK cells cytotoxicity could be a potential treatment target of GBM and improve patient prognosis.

In TME, the complement function is diverse, not only participating in anti-tumor defense but also promoting tumor progression and invasion. The two types combined body of $\mathrm{C} 3 \mathrm{a}$ and $\mathrm{C} 5 \mathrm{a}$ with their match receptors, $\mathrm{C} 3 \mathrm{aR}$ and $\mathrm{C} 5 \mathrm{aR} 1$ or $\mathrm{C} 5 \mathrm{aR} 2$, play a crucial role to in bringing about inflammation and cause activation of immune cells as well as specific malignant cells[34]. The influence that C3a and C5a exert on TME are the recruitment of tumor-promoting macrophages[35], and negative recruitment of CD4+ T cells, neutrophils[36] and NK cells[37]. PTX3 activates and modulates the complement cascade by the interaction with $\mathrm{C} 1 \mathrm{q}$ or Factor $\mathrm{H}$, and PTX3 involves in the recruitment of TAMs[35]. C5a recruit neutrophils and leads to the upregulation of receptors such as toll-like receptors (TLRs) and/or complement receptors. Ultimately this action could cause a more vigorous NET response[38]. Researchers illustrate that C3aR drives pro-tumoral neutrophil extracellular trap (NET) formation and the latter causes thrombus formation[39]. The embolus excites neutrophils into a protumorigenic (N2) state and strengthens the pathological process of hypercoagulation and alexin activation[39]. Finally, the interaction of the complement and the coagulation worse the outcome of GBM patients.

We found six immune-related genes were significantly correlated with prognosis and have a profound influence on the GBM microenvironment. In high-risk and low-risk groups, TAMs were positively relevant to the expression levels of prognostic IR-DEGs and have an impact on tumor progression and invasion. NK cells were negatively relevant to the prognostic IR-DEGs and enriched higher in low-risk group, resulting in a better prognosis. The differential activation of complement and coagulation cascade in the high-risk and low-risk groups were related to the prognostic IR-DEGs, which might explain the differential prognosis of GBM patients. They may become new strategies for improving GBM patient outcomes in the future.

In summary, we developed a novel prognostic risk score based on the expression of PLAUR, TNFSF14, CTSB, SOCS3, PTX3 and FCGR2B by using univariate and multivariate analysis in TCGA and CGGA databases. This prognostic signature can help predict the survival rate of GBM patients more accurately. What's more, we identified the difference of micro-environment in high- and low-risk group in GBM, and formed a regulatory network that needs further verification. These results provide a valuable insight into other further immunotherapy strategies, and the six prognostic IR-DEGs could become a target in improving the prognosis of GBM patients.

\section{Abbreviations}

GBM: glioblastoma; TCGA: The cancer genome atlas; CGGA: Chinese glioma genome atlas; GTEx: genotype-tissue expression; DEGs: differential genes; SRS: survival risk score; IR-DEGs: immune-related differential genes; TME: tumor microenvironment; OS: overall survival; TTF: tumor-treating fields; ICls: immune checkpoint inhibitors; PD-1: anti-programmed cell death protein-1; PD-L1: programmed death 
ligand-1; CTLA-4: anti-cytotoxic T lymphocyte-associated protein-4; TAM: tumor-associated macrophages; Tregs: regulatory $T$ cells.

\section{Declarations}

\section{Acknowledgements}

Not applicable.

\section{Funding}

This work was supported by the National Natural Science Foundation of China (NSFC) (No. 81703166), Natural Science Foundation of Guangdong Province (No. 2019A1515011943), China Postdoctoral Science Foundation (Nos. 2020T130052ZX and 2019M662974), Science and Technology Program of Guangzhou (Nos. 202002030445 and 202002030086), and Medical Scientific Research Foundation of Guangdong Province (Nos. A2020505, A2020499, B2021203, and B2021139). The funders had no role in study design, data analysis. All authors had full access to all the data in the study and had final responsibility for the decision to submit for publication.

\section{Availability of data and materials}

The datasets presented in this study can be found in online repositories. The names of the repository/repositories and accession number(s) can be found in the article.

\section{Ethics approval and consent to participate}

Not applicable.

\section{Competing interests}

The authors declare that they have no competing interests.

\section{Consent for publication}

Not applicable. 


\section{Author's contributions}

$Z Z, M L$ and JY designed the study. JY collected and analyzed data. JY and ZZ wrote the manuscript. All the authors approved the manuscript. All authors contributed to the article and approved the submitted version.

\section{Author details}

${ }^{1}$ Department of Oncology, Guangdong Sanjiu Brain Hospital, Guangzhou 510510, China. ${ }^{2}$ Department of Radiation Medicine, Guangdong Provincial Key Laboratory of Tropical Disease Research, School of Public Health, Southern Medical University, Guangzhou 510515, China. ${ }^{3}$ Department of Pathology, Guangdong Sanjiu Brain Hospital, Guangzhou 510510, China. ${ }^{4}$ Department of Radiation Oncology, Nanfang Hospital, Southern Medical University, Guangzhou 510515, China.

\section{References}

1. Tan AC, Ashley DM, López GY, Malinzak M, Friedman HS, Khasraw M: Management of glioblastoma: State of the art and future directions. CA Cancer J Clin 2020, 70(4):299-312.

2. Jiang T, Nam DH, Ram Z, Poon WS, Wang J, Boldbaatar D, Mao Y, Ma W, Mao Q, You Y et al: Clinical practice guidelines for the management of adult diffuse gliomas. CANCER LETT 2021, 499:60-72.

3. Campos B, Olsen LR, Urup T, Poulsen HS: A comprehensive profile of recurrent glioblastoma. ONCOGENE 2016, 35(45):5819-5825.

4. Wang X, Guo G, Guan H, Yu Y, Lu J, Yu J: Challenges and potential of PD-1/PD-L1 checkpoint blockade immunotherapy for glioblastoma. J Exp Clin Cancer Res 2019, 38(1):87.

5. Huang S, Song Z, Zhang T, He X, Huang K, Zhang Q, Shen J, Pan J: Identification of Immune Cell Infiltration and Immune-Related Genes in the Tumor Microenvironment of Glioblastomas. FRONT IMMUNOL 2020, 11:585034.

6. Schmidt MC, Antweiler S, Urban N, Mueller W, Kuklik A, Meyer-Puttlitz B, Wiestler OD, Louis DN, Fimmers R, von Deimling A: Impact of genotype and morphology on the prognosis of glioblastoma. $J$ Neuropathol Exp Neurol 2002, 61(4):321-328.

7. Delgado-López PD, Corrales-García EM: Survival in glioblastoma: a review on the impact of treatment modalities. CLIN TRANSL ONCOL 2016, 18(11):1062-1071.

8. Sokratous G, Polyzoidis S, Ashkan K: Immune infiltration of tumor microenvironment following immunotherapy for glioblastoma multiforme. Hum Vaccin Immunother 2017, 13(11):2575-2582.

9. Quail DF, Joyce JA: The Microenvironmental Landscape of Brain Tumors. CANCER CELL 2017, 31(3):326-341.

10. Salmaggi A, Eoli M, Frigerio S, Silvani A, Gelati M, Corsini E, Broggi G, Boiardi A: Intracavitary VEGF, bFGF, IL-8, IL-12 levels in primary and recurrent malignant glioma. J Neurooncol 2003, 62(3):297-303. 
11. Agliardi G, Liuzzi AR, Hotblack A, De Feo D, Núñez N, Stowe CL, Friebel E, Nannini F, Rindlisbacher L, Roberts TA et al: Intratumoral IL-12 delivery empowers CAR-T cell immunotherapy in a pre-clinical model of glioblastoma. NAT COMMUN 2021, $12(1): 444$.

12. Roumenina LT, Daugan MV, Petitprez F, Sautès-Fridman C, Fridman WH: Context-dependent roles of complement in cancer. NAT REV CANCER 2019, 19(12):698-715.

13. Reis ES, Mastellos DC, Ricklin D, Mantovani A, Lambris JD: Complement in cancer: untangling an intricate relationship. NAT REV IMMUNOL 2018, 18(1):5-18.

14. Zha H, Wang X, Zhu Y, Chen D, Han X, Yang F, Gao J, Hu C, Shu C, Feng Y et al: Intracellular Activation of Complement C3 Leads to PD-L1 Antibody Treatment Resistance by Modulating Tumor-Associated Macrophages. CANCER IMMUNOL RES 2019, 7(2):193-207.

15. Bouwens VDVT, Kros JM, Mustafa D, van Wijck R, Ackermans L, van Hagen PM, van der Spek PJ: The complement system in glioblastoma multiforme. Acta Neuropathol Commun 2018, 6(1):91.

16. Bouwens TA, Trouw LA, Veerhuis R, Dirven CM, Lamfers ML, Al-Khawaja H: Complement activation in Glioblastoma multiforme pathophysiology: evidence from serum levels and presence of complement activation products in tumor tissue. J NEUROIMMUNOL 2015, 278:271-276.

17. Zhang X, Lan Y, Xu J, Quan F, Zhao E, Deng C, Luo T, Xu L, Liao G, Yan M et al: CellMarker: a manually curated resource of cell markers in human and mouse. NUCLEIC ACIDS RES 2019, 47(D1):D721D728.

18. Newman AM, Liu CL, Green MR, Gentles AJ, Feng W, Xu Y, Hoang CD, Diehn M, Alizadeh AA: Robust enumeration of cell subsets from tissue expression profiles. NAT METHODS 2015, 12(5):453-457.

19. Louis DN, Perry A, Reifenberger G, von Deimling A, Figarella-Branger D, Cavenee WK, Ohgaki $H$, Wiestler OD, Kleihues P, Ellison DW: The 2016 World Health Organization Classification of Tumors of the Central Nervous System: a summary. ACTA NEUROPATHOL 2016, 131(6):803-820.

20. Lacroix M, Abi-Said D, Fourney DR, Gokaslan ZL, Shi W, DeMonte F, Lang FF, McCutcheon IE, Hassenbusch SJ, Holland E et al: A multivariate analysis of $\mathbf{4 1 6}$ patients with glioblastoma multiforme: prognosis, extent of resection, and survival. J NEUROSURG 2001, 95(2):190-198.

21. Fiorica F, Colella M, Taibi R, Bonetti A, Giuliani J, Perrone MS, Missiroli S, Giorgi C: Glioblastoma: Prognostic Factors and Predictive Response to Radio and Chemotherapy. CURR MED CHEM 2020, 27(17):2814-2825.

22. Huang P, Li L, Qiao J, Li X, Zhang P: Radiotherapy for glioblastoma in the elderly: A protocol for systematic review and meta-analysis. Medicine (Baltimore) 2020, 99(52):e23890.

23. Aldape K, Zadeh G, Mansouri S, Reifenberger G, von Deimling A: Glioblastoma: pathology, molecular mechanisms and markers. ACTA NEUROPATHOL 2015, 129(6):829-848.

24. Eckel-Passow JE, Lachance DH, Molinaro AM, Walsh KM, Decker PA, Sicotte H, Pekmezci M, Rice T, Kosel ML, Smirnov IV et al: Glioma Groups Based on 1p/19q, IDH, and TERT Promoter Mutations in Tumors. N Engl J Med 2015, 372(26):2499-2508.

25. Ohgaki $\mathrm{H}$, Kleihues $\mathrm{P}$ : The definition of primary and secondary glioblastoma. CLIN CANCER RES 2013, 19(4):764-772. 
26. Klemm F, Maas RR, Bowman RL, Kornete M, Soukup K, Nassiri S, Brouland JP, lacobuzio-Donahue CA, Brennan C, Tabar V et al: Interrogation of the Microenvironmental Landscape in Brain Tumors Reveals Disease-Specific Alterations of Immune Cells. CELL 2020, 181(7):1643-1660.

27. Hambardzumyan $\mathrm{D}$, Gutmann $\mathrm{DH}$, Kettenmann $\mathrm{H}$ : The role of microglia and macrophages in glioma maintenance and progression. NAT NEUROSCI 2016, 19(1):20-27.

28. Shi C, Pamer EG: Monocyte recruitment during infection and inflammation. NAT REV IMMUNOL 2011, 11(11):762-774.

29. Markovic DS, Vinnakota K, Chirasani S, Synowitz M, Raguet H, Stock K, Sliwa M, Lehmann S, Kälin R, van Rooijen $\mathrm{N}$ et al: Gliomas induce and exploit microglial MT1-MMP expression for tumor expansion. Proc Natl Acad Sci U S A 2009, 106(30):12530-12535.

30. Lehnardt S: Innate immunity and neuroinflammation in the CNS: the role of microglia in Toll-like receptor-mediated neuronal injury. GLIA 2010, 58(3):253-263.

31. Hu F, Ku MC, Markovic D, Dzaye O, Lehnardt S, Synowitz M, Wolf SA, Kettenmann H: Gliomaassociated microglial MMP9 expression is upregulated by TLR2 signaling and sensitive to minocycline. INT J CANCER 2014, 135(11):2569-2578.

32. Levy EM, Roberti MP, Mordoh J: Natural killer cells in human cancer: from biological functions to clinical applications. J Biomed Biotechno/2011, 2011:676198.

33. Shimasaki N, Jain A, Campana D: NK cells for cancer immunotherapy. NAT REV DRUG DISCOV2020, 19(3):200-218.

34. Merle NS, Noe R, Halbwachs-Mecarelli L, Fremeaux-Bacchi V, Roumenina LT: Complement System Part II: Role in Immunity. FRONT IMMUNOL 2015, 6:257.

35. Bonavita E, Gentile S, Rubino M, Maina V, Papait R, Kunderfranco P, Greco C, Feruglio F, Molgora M, Laface I et al: PTX3 is an extrinsic oncosuppressor regulating complement-dependent inflammation in cancer. CELL 2015, 160(4):700-714.

36. Nabizadeh JA, Manthey HD, Steyn FJ, Chen W, Widiapradja A, Md AF, Boyle GM, Taylor SM, Woodruff TM, Rolfe BE: The Complement C3a Receptor Contributes to Melanoma Tumorigenesis by Inhibiting Neutrophil and CD4+ T Cell Responses. J IMMUNOL 2016, 196(11):4783-4792.

37. Janelle V, Langlois MP, Tarrab E, Lapierre P, Poliquin L, Lamarre A: Transient complement inhibition promotes a tumor-specific immune response through the implication of natural killer cells. CANCER IMMUNOL RES 2014, 2(3):200-206.

38. de Bont CM, Boelens WC, Pruijn G: NETosis, complement, and coagulation: a triangular relationship. CELL MOL IMMUNOL 2019, 16(1):19-27.

39. Guglietta S, Chiavelli A, Zagato E, Krieg C, Gandini S, Ravenda PS, Bazolli B, Lu B, Penna G, Rescigno $\mathrm{M}$ : Coagulation induced by C3aR-dependent NETosis drives protumorigenic neutrophils during small intestinal tumorigenesis. NAT COMMUN2016, 7:11037.

\section{Figures}




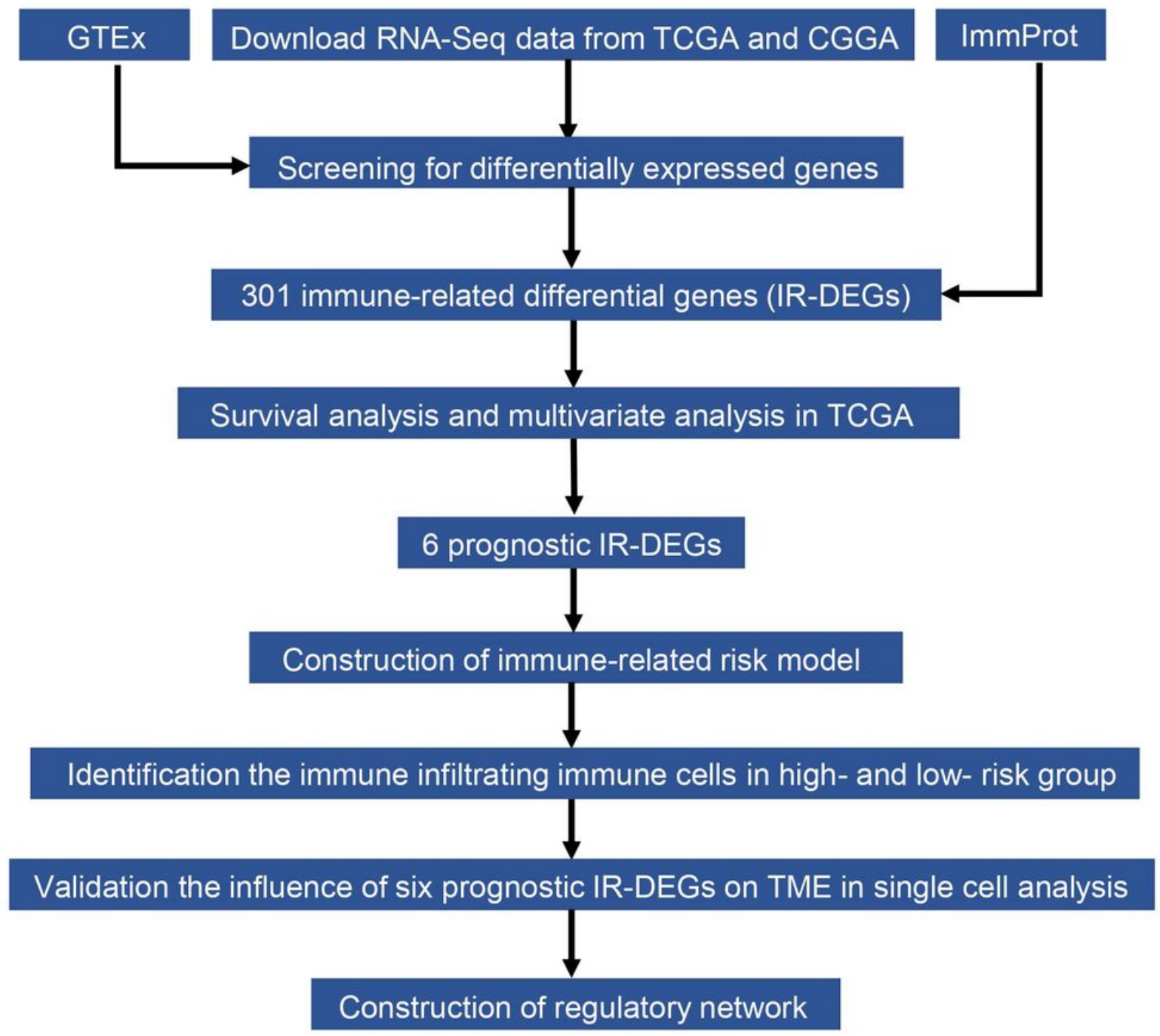

Figure 1

Workflow of the whole analysis 
a

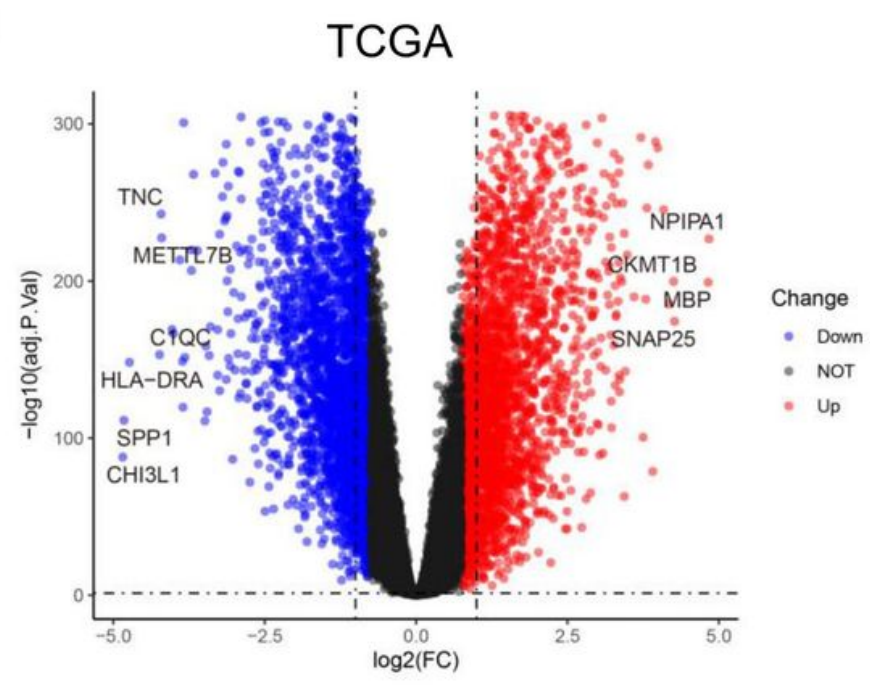

b
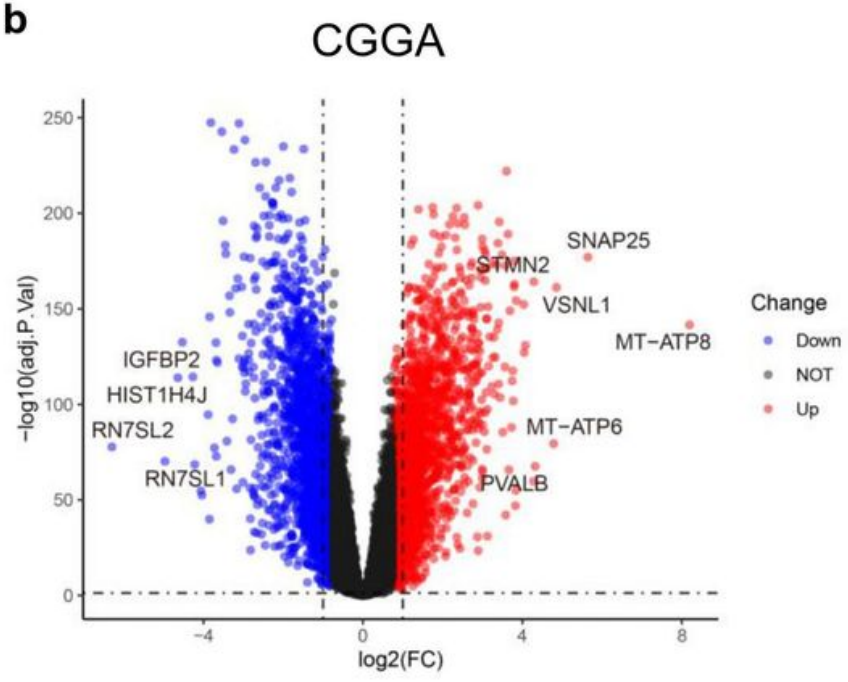

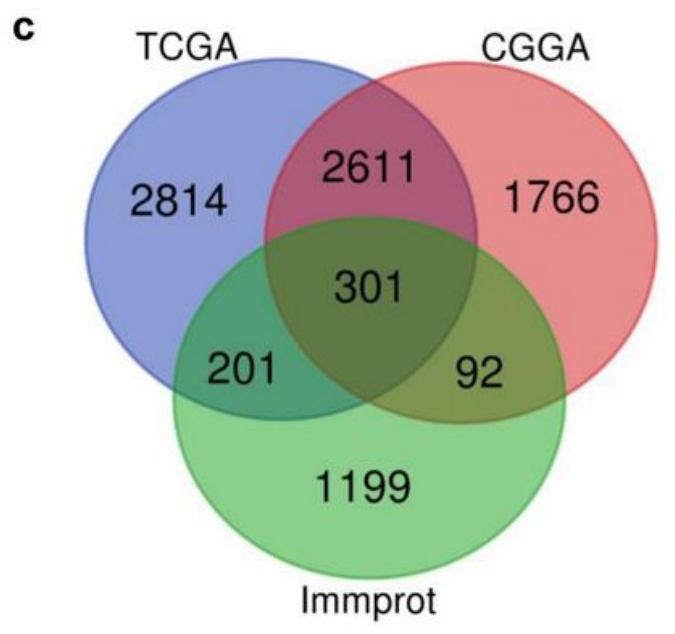

Figure 2

Identification of IR-DEGs. (a) Volcano plot of differential genes in TCGA. (b) Volcano plot of differential genes in CGGA. (c) Venn diagram of differential genes and immune-related genes in ImmProt database. 
a

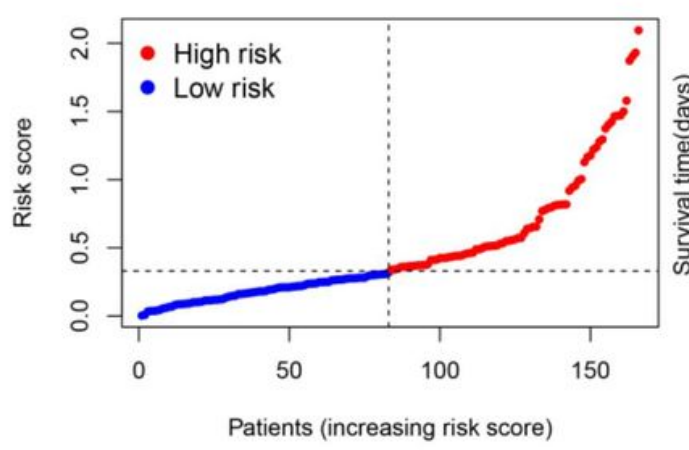

b

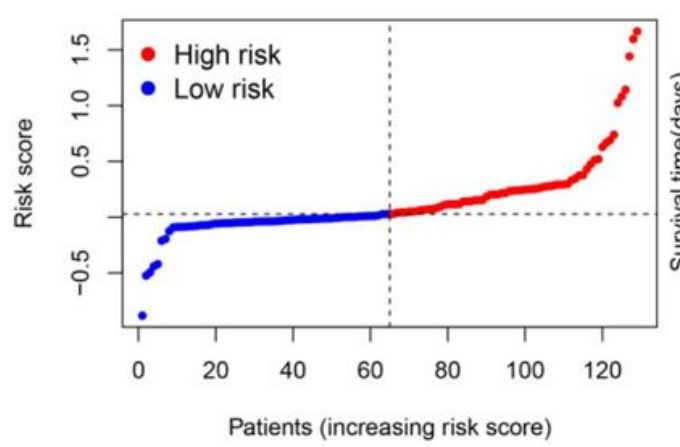

TCGA
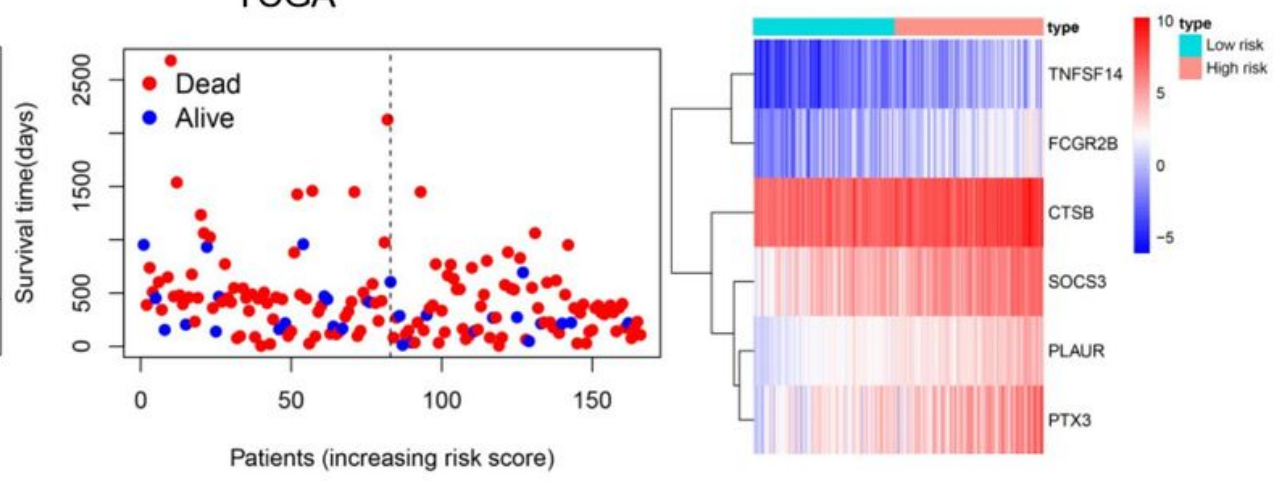

CGGA
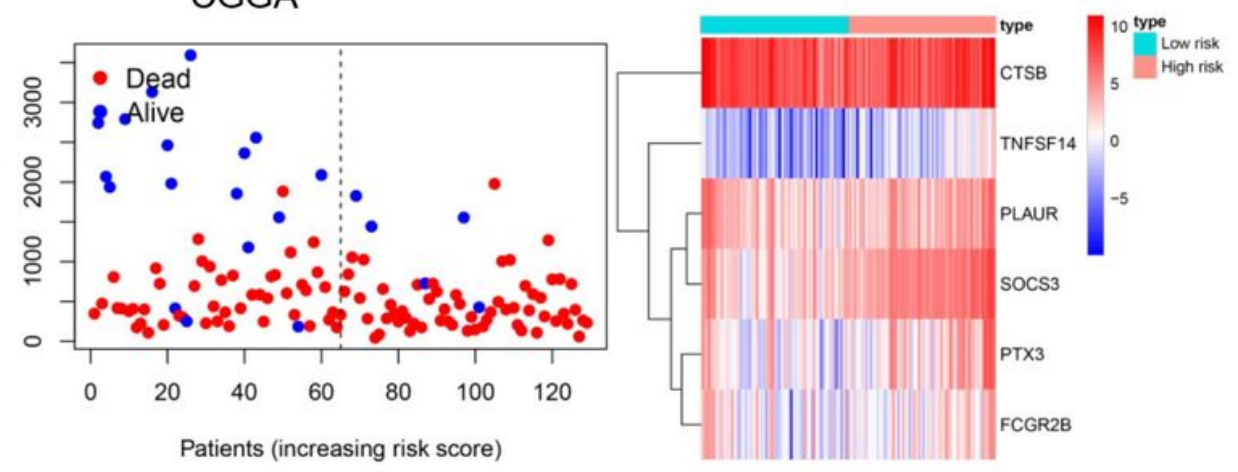

d

CGGA

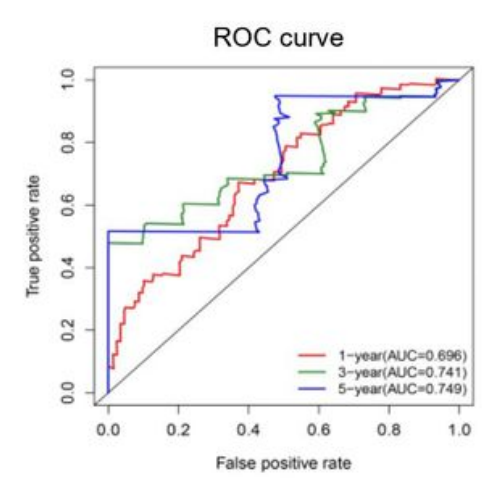

TCGA
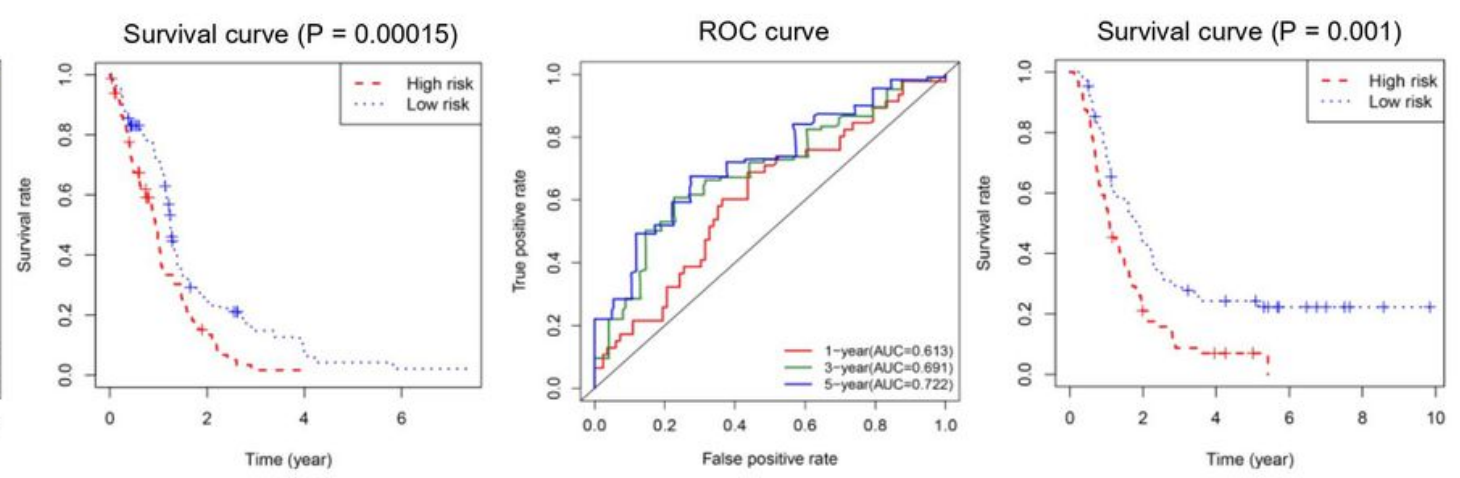

\section{Figure 3}

Evaluating the prognostic of risk score. Analysis of risk score in TCGA testing set (a) and CGGA validation cohort (b). The first from left represents the values of risk score in each patient. Red points are samples with higher risk score, and blue points are samples with lower risk score. The intersection of the two dashed lines represents the median value of risk score. Second from left shows the distribution of survival status. Heatmap shows the expression level of six prognostic genes. Kaplan Meier survival analysis was performed to predict the OS of low- and high- patients in TCGA (c) and CGGA (d). Time- 
dependent ROC curve shows the evaluation of risk score for predicting 1-year, 3-year and 5-year in TCGA and CGGA dataset.
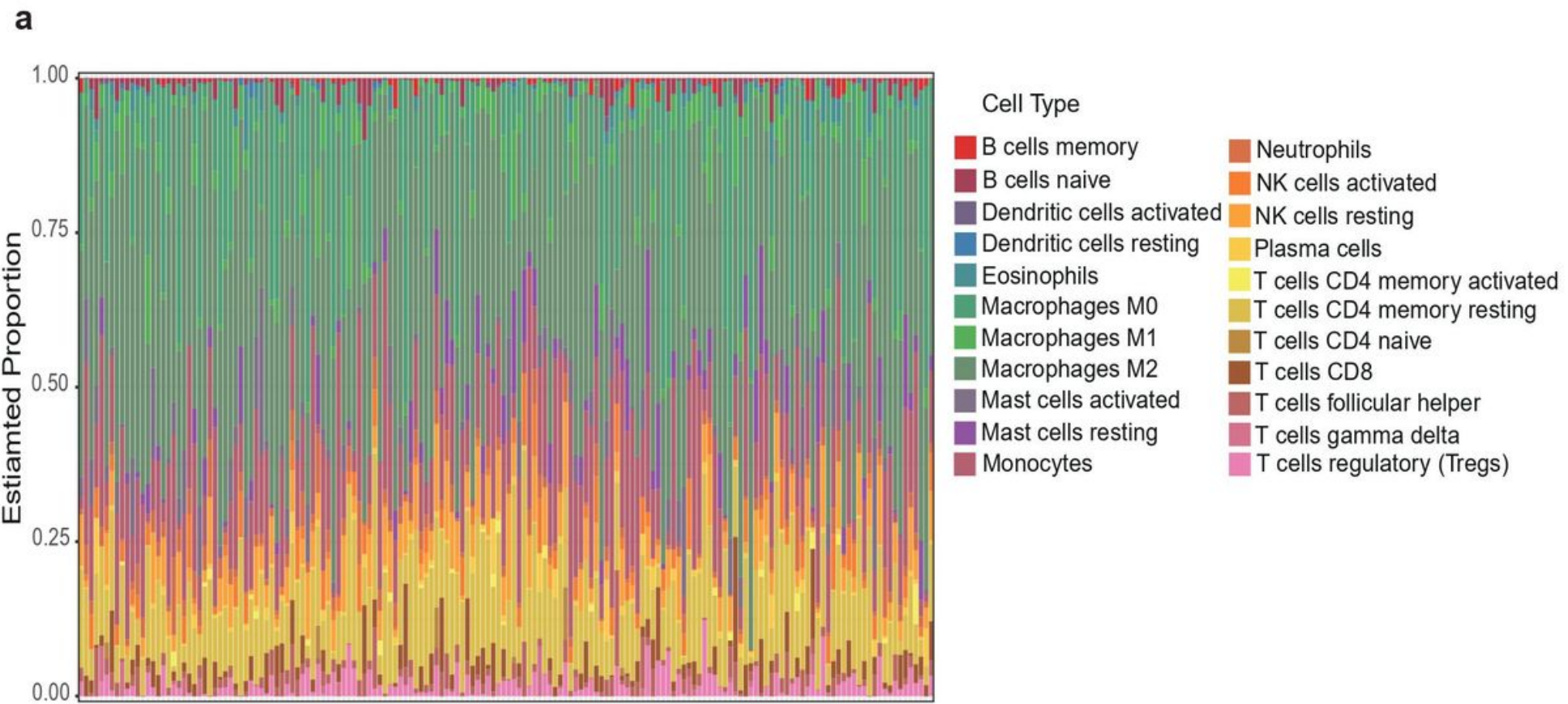

b

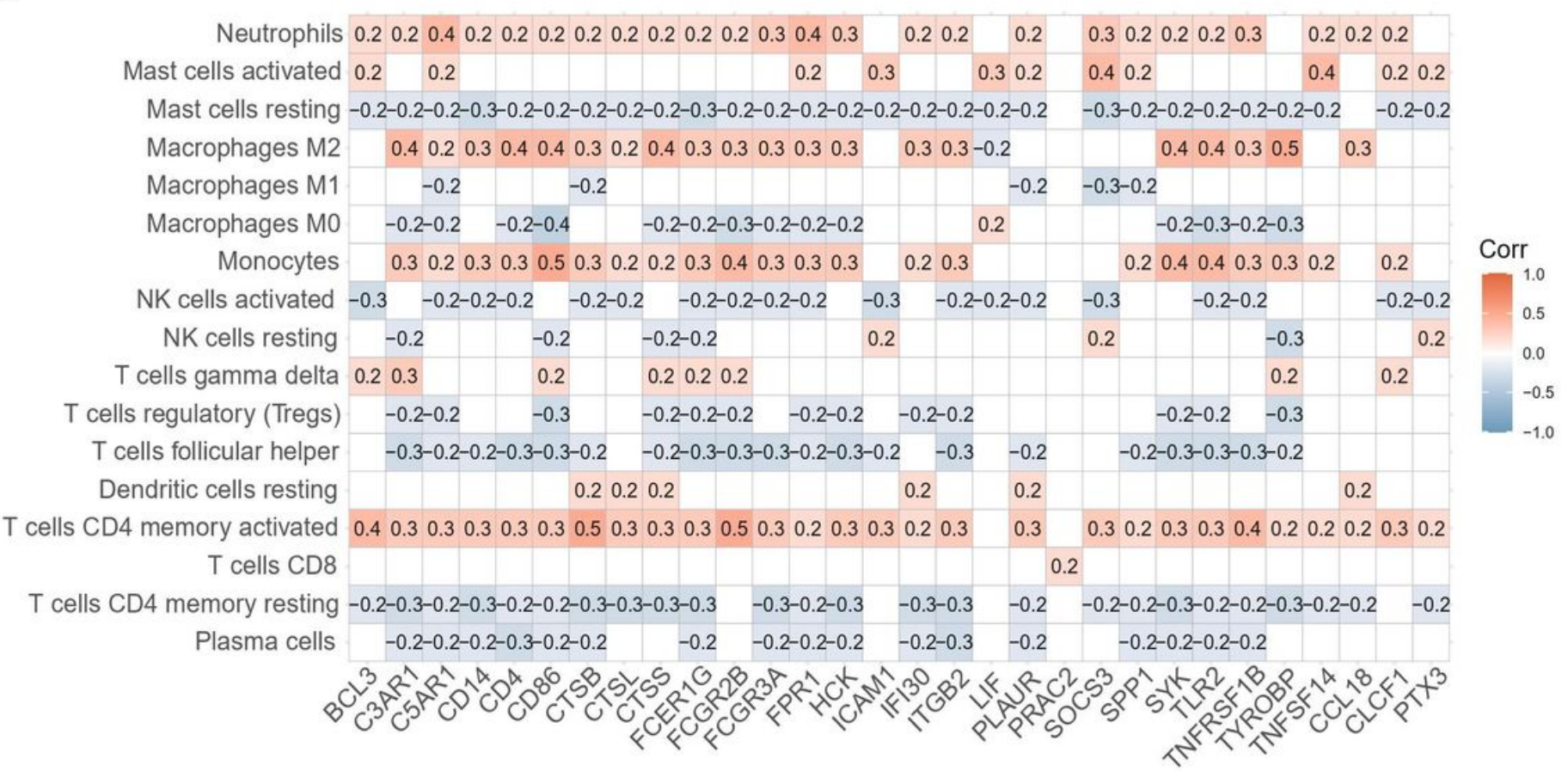

\section{Figure 4}

Tumor-Infiltrating Immune Cells in high- and low- risk group. (a) Bar plot indicated the difference infiltrating immune cell type distribution between high-risk and low-risk group. (b) Corheatmap showed correlation of tumor infiltrating immune cell type and the expression of six prognostic genes and their coexpressed genes in GBM. 
a

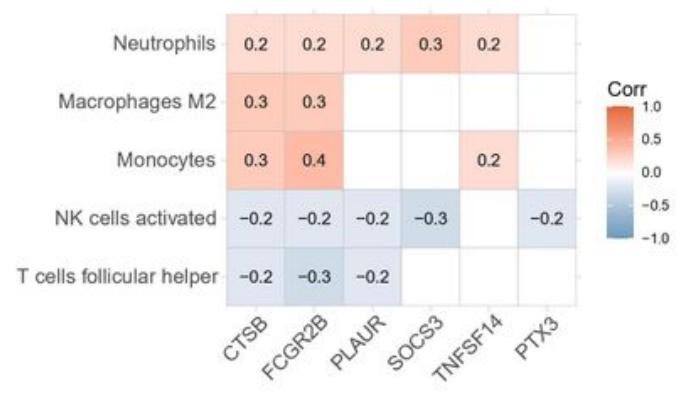

b

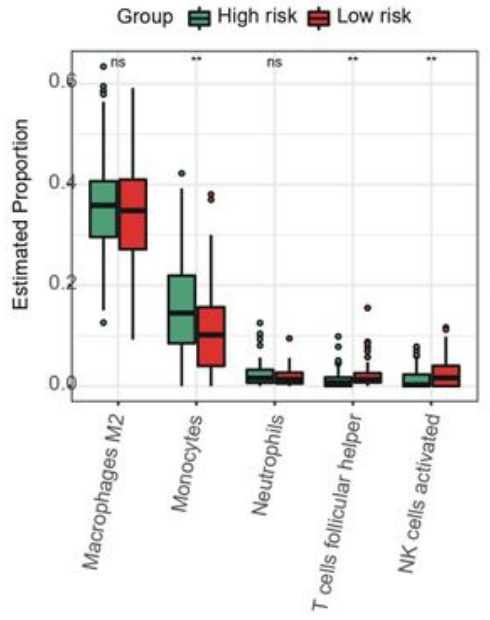

c
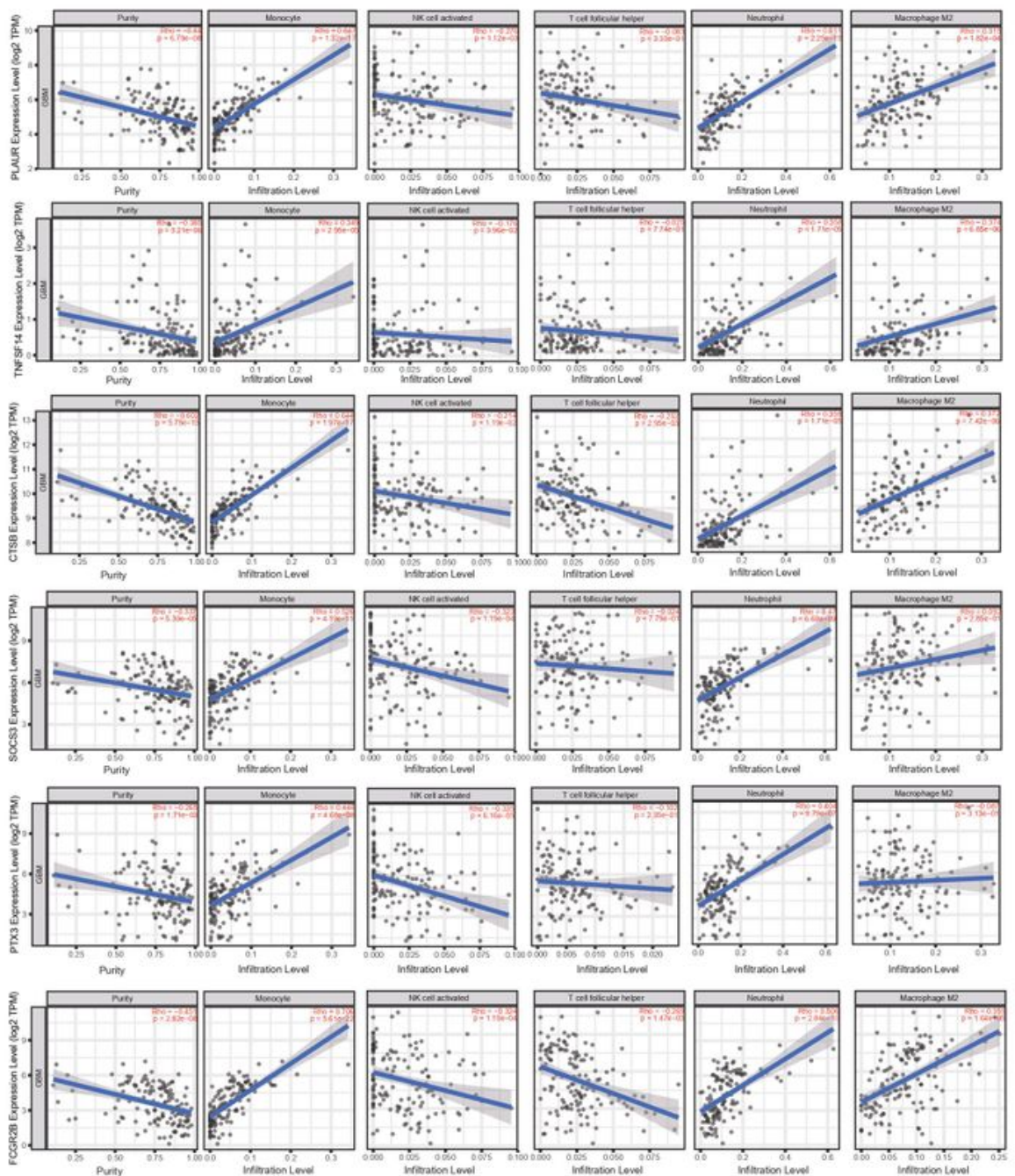

\section{Figure 5}

Relationship between prognostic IR-DEGs and tumor infiltrating immune cell. (a) The correlation between prognostic genes and significant tumor infiltrating immune cells. (b) Box plot of the difference of significant infiltrating immune cells in high- and low-risk group. (c) The correlation analysis between the six prognostic genes and significant tumor infiltrating immune cells was performed in Timer. 


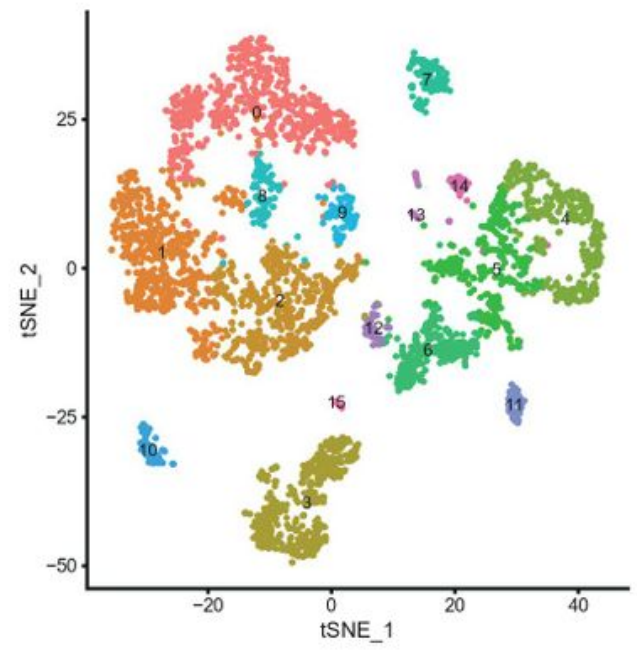

C

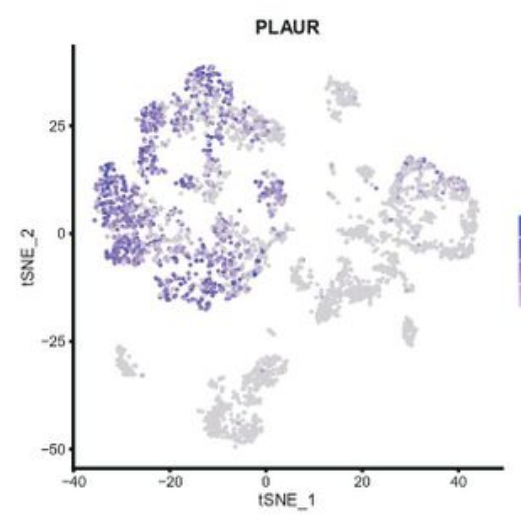

d

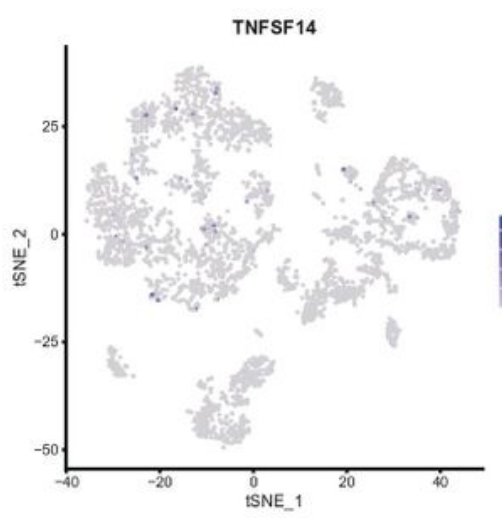

g

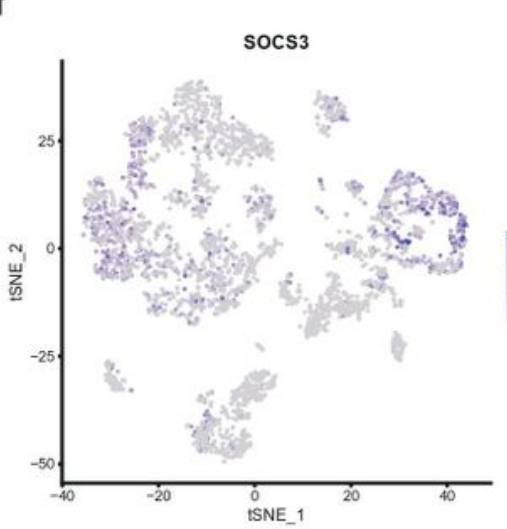

b

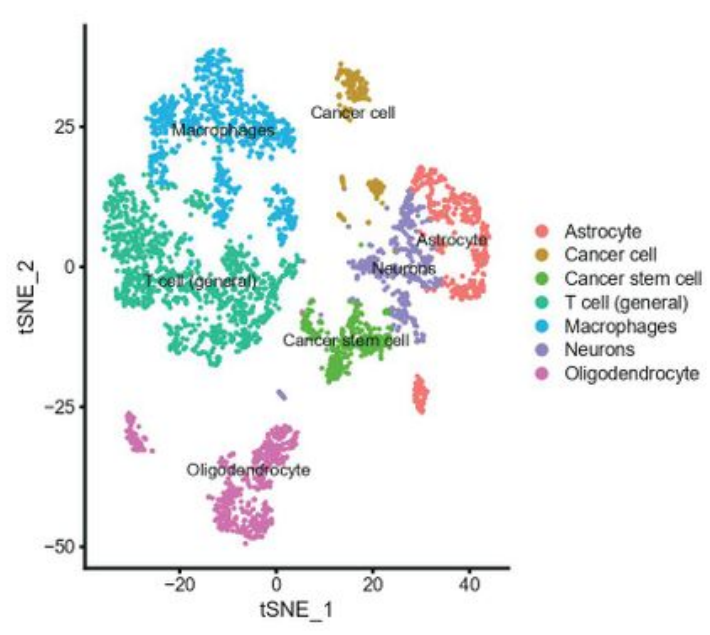

e

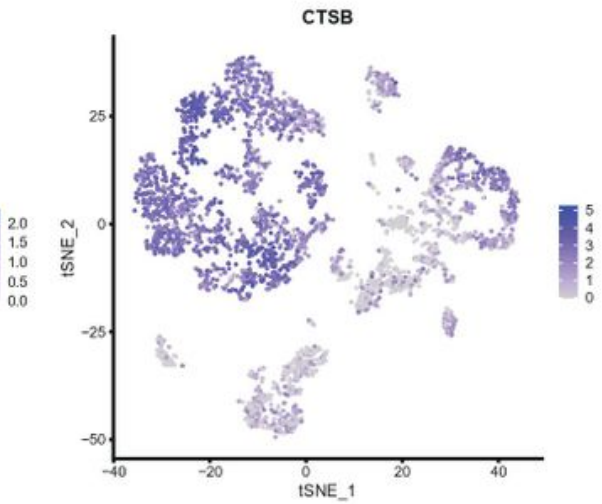

h

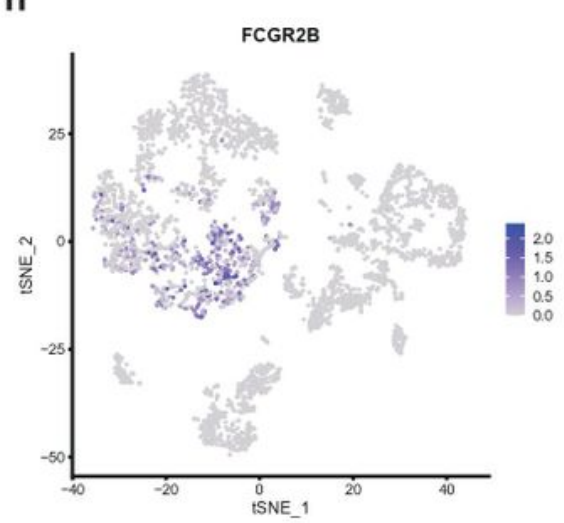

\section{Figure 6}

Validation the influence of prognostic IR-DEGs on TME. (a) t-distributed neighbor embedding(tSNE) plot of all single cells, and divided into 15 clusters. (b) 15 clusters were annotated by singleR package and CellMarker according to the composition of the marker genes. (c-h) Expression of six prognostic genes in 15 clusters. 
a

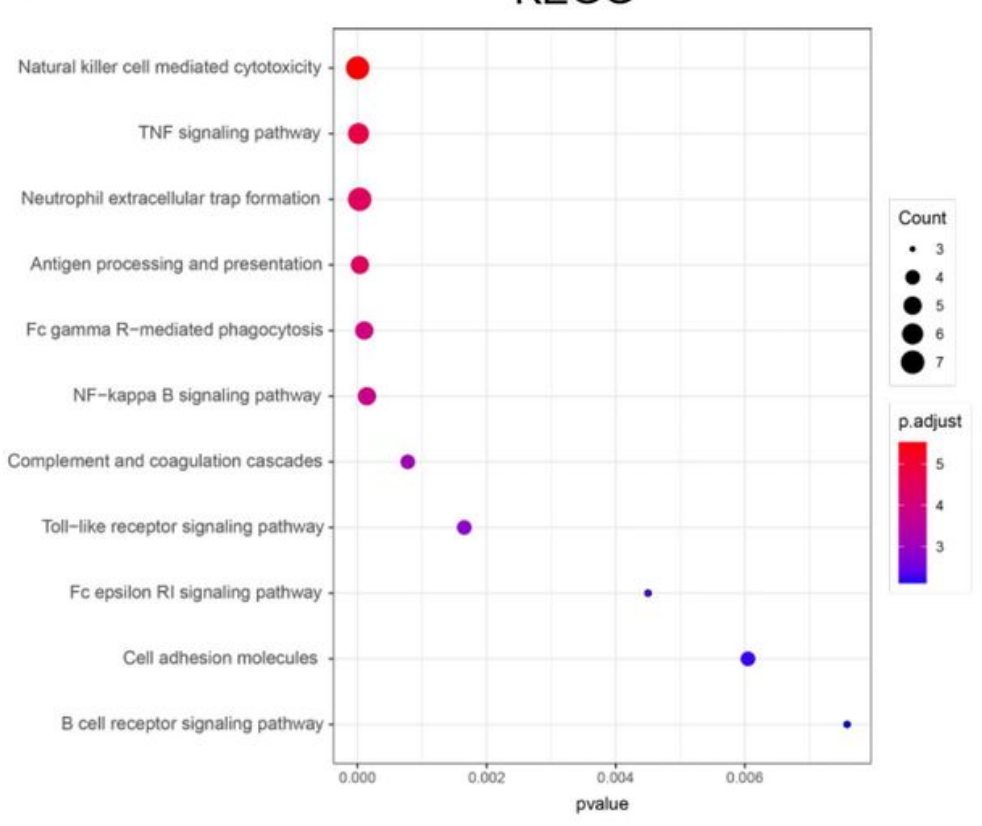

b

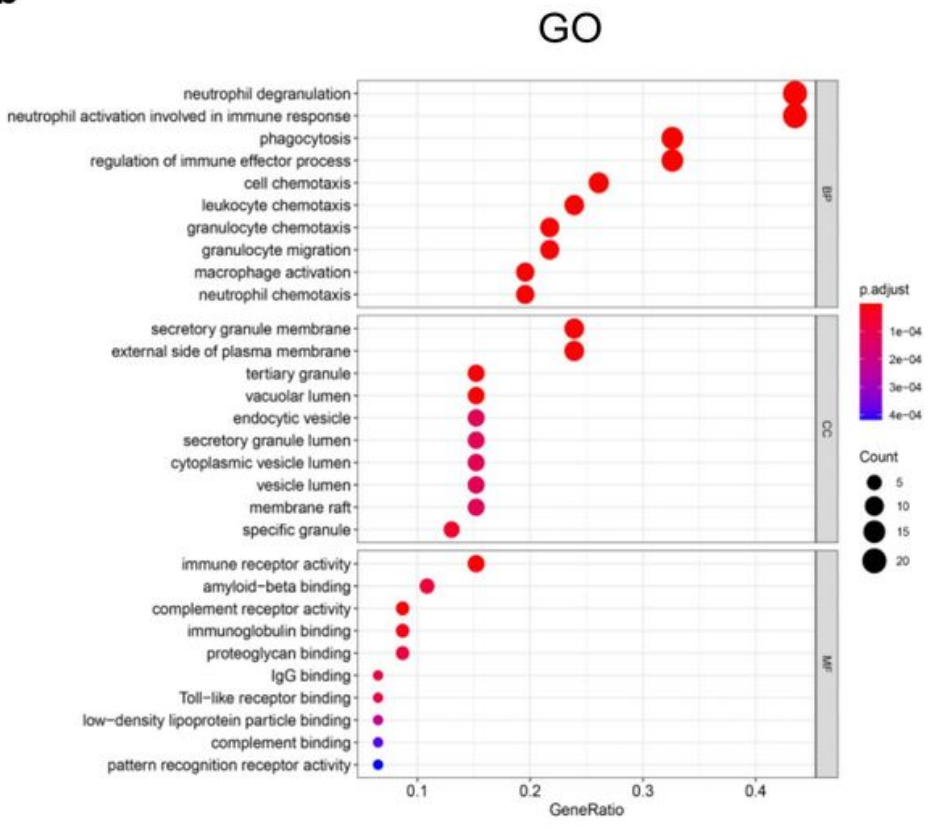

Figure 7

Functional enrichment analysis of IR-DEGs and their co-expressed genes. (a) KEGG pathways analysis.

(b) Go enrichment analysis. 
a

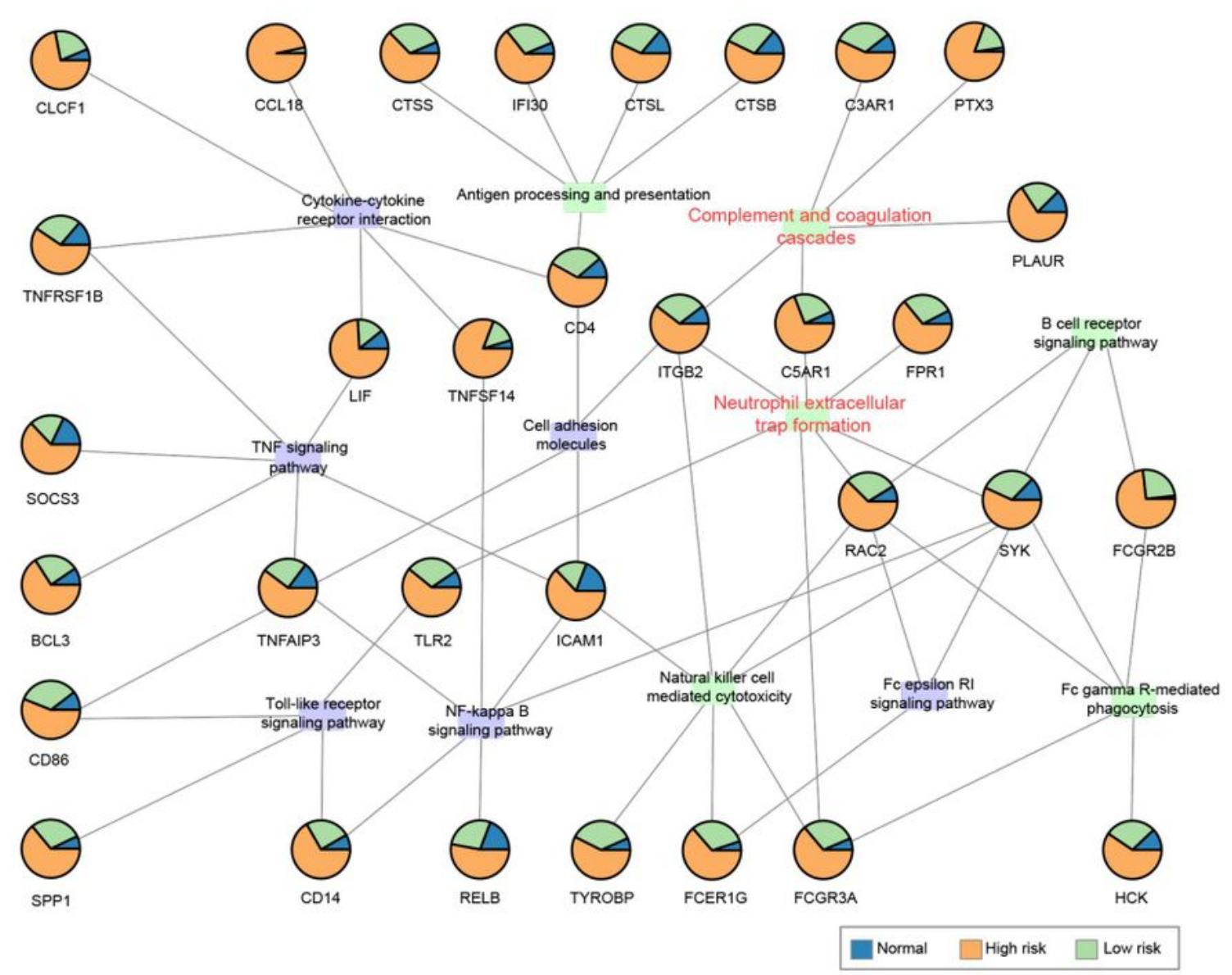

b

\section{Figure 8}

Regulatory network of IR-DEGs. (a) Regulatory network of IR-DEGs and activated pathways. (b) Regulatory network of IR-DEGs and transcription factors. 